DR. IRVINE: Fundamentally, from our discussion about priorities it follows that this is an expensive habit and causes a lot of human misery-bronchitis, lung cancer, and heart disease. We're now in the position when we know the causes of some of the major degenerative diseases; what are we going to do about these?

CHAIRMAN: Another question we ought to consider briefly is how much should the medical profession resist measures that are thrust on them by public pressure-such as cervical smears or abortion on demand.

PROFESSOR RHODES: One had some sympathy with the Abortion Act and our figures for abortions were beginning to increase before the issue became a political one.

DR. IRVINE: This raises the question whether abortion was really imposed on the profession: I doubt whether it is possible to impose anything on any articulate body of professional people unless they are already half-consulted or persuaded.

PROFESSOR RHODES: That's true, but I would like to grind an axe; the Bill was utterly wrong in citing four categories for abortion. There is no other area in medicine where the indications for an operation are spelt out and where you have to notify operations. If there is ever a clash between the interest of medicine and of society, it's the professional's job to see that we don't get the specificity that ran into the act, which hindered freedom of interpretation very greatly.

DR. IRVINE: Are we happy with the way doctors communicate with society at the moment.

DR. OGG: No, I'm not; I don't think that society really knows our problems and the implications of cutting the cake in a particular way. If a decision is made by government to restrict availability of dialysis, this should be stated clearly and publicly, so that the individual doctor does not have to make lame excuses to the relatives of his dying patient.

DR. IRVINE : I wonder whether as a profession we spend enough time in public debate on vital issues? We don't, for instance, say very much about how people should use this very complicated health service.

PROFESSOR RHODES: We have no suitable accountancy; we have to measure everything in terms of money and yet we're all well aware of social implications. What we need is "social accountancy" with appropriate weighting. The best parallel to this is the purely economic brief given to Lord Beeching when he was asked to look at the future of the railways. Within his brief he worked very well; he closed railways and we could slash medicine in many ways, but we have always known that economic considerations aren't the only ones.

New Appliances

\title{
Evaluation of the new "Williams" Anaesthetic Filter
}

British Medical fournal, 1973, 2, 653-654

Dr. N. J. MITCHELL, senior microbiologist at the Public Health Laboratory, Epsom, and Dr. D. R. GAMBLE, director of the Public Health Laboratory, Epsom, write: Effective decontamination of complex anaesthetic equipment and mechanical ventilators has proved time-consuming, difficult, and sometimes hazardous. The idea of protecting equipment from bacterial contamination from the patient by the interposition of a filter seemed likely to circumvent the problem. In practice, however, filters were found to absorb water from the patient's expired air, producing an unacceptable increase in resistance to airflow, and bacterial aerosols readily penetrated the water logged filter. ${ }^{\text {l }}$ Various attempts have been made to solve this condensation problem such as using disposable filters ${ }^{2}$ and enclosing the filter in an electrically heated mantle. ${ }^{3}$ More recently a siliconized filter element has been marketed which appears to be sufficiently water-repellent to prevent appreciable water absorption. ${ }^{4}$ Inquiries disclosed that many of our colleagues were unaware that the improved filter was available, while others were using the filter and were satisfied with its performance, although no published results of satisfactory bacteriological tests were available.

\section{Performance}

\section{BACTERIOLOGICAL TESTS}

The filter unit was fitted in the expiratory circuit of an anaesthetic machine, but after prolonged usage in a busy surgical theatre it was found that swabs taken from both input and output ports yielded no bacterial growth.
The only opportunity for a satisfactory in-use test occurred when a deeply comatose patient required mechanical ventilation. After four days swabs taken from the trachea, the $Y$ piece of the expiratory valve, and the filter input port yielded a heavy growth of Klebsiella aerogenes. The output port of the filter and the expiratory port of the ventilator remained sterile and there was no measurable increase in resistance to airflow. It was clear that the bacterial challenge met by the filter during routine use would be occasional and unpredictable and might fail to show deficiences in the filter. A more stringent laboratory procedure was therefore devised.

An overnight nutrient broth culture of Serratia marcescens was suspended in quarter-strength Ringer's solution to contain $10^{9}$ organisms per $\mathrm{ml}$. An aerosol produced from this suspension in a DeVilbis nebulizer was discharged at a rate of 10 litres per minute into the input port of the filter. The output port was directed into the air intake of a Casella slit-sampler which was set to sample at a rate of 700 litres of air per minute on to a $13-\mathrm{cm}$ nutrient agar plate. A total of 14,000 litres of heavily contaminated moist air was passed through the filter. The culture plates were incubated aerobically overnight at $37^{\circ} \mathrm{C}$ and at room temperature for a further 24 hours. All the plates were sterile. Swabs taken from the input port of the filter unit and inoculated on nutrient agar produced a heavy growth of $S$. marcescens. The output port was sterile. After removal of the filter a control plate exposed to 350 litres of the aerosol yielded uncountable colonies of $S$. marcescens.

To check the efficiency of the silicone treatment the filter was exposed to a four-hour continuous flow of a tap-water aerosol discharged direct into the input port at a rate of 10 litres per minute. This was followed by 3,500 litres of heavily contaminated air as described above. The slit-sample culture plate was sterile, while the control plate yielded a heavy 
growth. Again swabs from the input port yielded a heavy growth of $S$. marcescens. The output port was sterile.

\section{STERILIZATION}

The manufacturers state that the unit can be sterilized in a high-vacuum autoclave. To confirm this the filter unit was left in a high-vacuum autoclave for one week during which it was subjected to 110 successive $134^{\circ} \mathrm{C}$ sterilizing cycles. The entire range of experiments described above were then repeated and the bacteriological results were identical.

\section{FILTER RESISTANCE}

Resistance was measured in centimetres of water at airflow rates of 15 litres and 45 litres per minute after the filter had been exposed to a water aerosol for four hours. There was no measurable increase in resistance.

\section{SODIUM CHLORIDE FLAME TEST}

At the conclusion of all these experiments the filter was subjected to the sodium flame test procedure in accordance with British Standard 3928. It achieved a result better than $0.001 \%$ where the flow rate through the filter was 62.5 litres per minute.

\section{Conclusions}

Siliconization of the filter medium appears to have solved the problem of water saturation and represents an important advance in filter design. The experiments performed on the new Williams anaesthetic filter after prolonged exposure to a water aerosol failed to show loss of bacterial filtering efficiency or increase in airflow resistance. The efficiency of the filter remained unimpaired after subjection to 110 autoclave sterilizing cycles.

Such a filter placed between the patient and a mechanical ventilator or anaesthetic equipment should eliminate the need for decontamination. Over a period of time, however, it is possible that small particles of debris and mucus might accumulate within the interstices of the filter material, increasing its resistance to airflow. For this reason the manufacturer recommends that the filter be returned for yearly servicing. It is, however, reassuring that none of the filters returned for servicing has shown any increase in airflow resistance.

The Williams anaesthetic and respiratory filter is available from Foramaflow Ltd., Frimley, Surrey.

We acknowledge the help given by Mr. D. Lowe, of Foramaflow, who performed the sodium flame test.

\section{References}

1 Hellewell, J., Jeanes, A. L., Watkins, R. R., and Gibbs, T. J., Anaesthesia, 1967 22, 497.

Martin J. T., and Ulrich, J. A., Anaesthesia and Analgesia, 1969, 48, 944.

3 Pyke, P., Darlow, M.. and Firman, J. E., Lancet, 1969, 1, 136.

4 Lowe, D., Hospital Engineering, 1971, 25, 171.

\section{Reorganization-I974 or 1984?}

\section{The Metropolitan District of Gateshead}

\section{FROM A SPECIAL CORESPONDENT}

\section{British Medical fournal, 1973, 2, 654-655}

Though 94,680 people now live in Gateshead (south of the river Tyne from Newcastle) from 1974 for health and local government purposes it will more than double in size by taking in surrounding areas which up to now have been in County Durham. Dr. Gyles Riddle, a general practitioner in Gateshead, and member of the General Medical Services Committee of the B.M.A., told me what the implications of this change were. To start with, the No. 1 Regional Health Authority would be responsible for all branches of medicine in the Newcastle region. The Region would be subdivided into nine areas, of which the new Gateshead would be one. But Gateshead itself would not be further subdivided into districts-in other words, it would form a single district area.

Dr. Riddle was at pains to emphasize the differences between the terms used in the local authority reorganization and those used for the new N.H.S. In local authority terms TyneWear would be a metropolitan county, itself divided into five metropolitan districts. Each of these local authority metropolitan districts would be coterminous with an N.H.S. area, administered by an area health authority. The area health authorities which corresponded to metropolitan districts, both in Newcastle and elsewhere, would usually be "areas-without-districts." To confuse matters further, the word "district" crops up again in local authority terms, because their non-metropolitan counties are to have district councils. These may or may not be coterminous with an N.H.S. district. In such a county the local authority will provide social services which may be divided into districts which are not necessarily coterminous with N.H.S. districts. "The only thing you can clutch at," Dr. Riddle concluded, "is that the area health authority is coterminous with its local authority." This was, however, an advance on the present arrangements.

So the new metropolitan district of Gateshead will have an area health authority, with a single area management team. The new population will be about 220,000 and will therefore correspond fairly closely to the average National Health Service district described in the "Grey Book." Dr. Riddle thought that the needs outlined for such a district were probably about right for Gateshead.

\section{Method of Election}

How were the authorities going to be appointed, I asked. Some thought the members were to be elected, but this was not true, Dr. Riddle said. The chairman and the members of each regional authority were to be appointed by the Secretary of State. At the area level, doctors and nurses would be among the members; four members would be appointed by the corresponding local authority (probably from those who took an interest in the social services) and the remainder, including the chairman, by the regional 